\title{
Nomogram prediction for the overall survival and cancer-specific survival of patients diagnosed with Merkel cell carcinoma
}

\author{
Xufeng Yin^, Huihui She, Lorna Martin Kasyanju Carrero, Weiwei Ma, Bingrong Zhou \\ Department of Dermatology, the First Affiliated Hospital of Nanjing Medical University, Nanjing, China \\ Contributions: (I) Conception and design: B Zhou, X Yin; (II) Administrative support: B Zhou; (III) Provision of study materials or patients: B Zhou, X \\ Yin, H She; (IV) Collection and assembly of data: X Yin, L Martin Kasyanju Carrero, W Ma; (V) Data analysis and interpretation: L Martin Kasyanju \\ Carrero, W Ma, H She; (VI) Manuscript writing: All authors; (VII) Final approval of manuscript: All authors. \\ Correspondence to: Bingrong Zhou, MD, PhD. Department of Dermatology, The First Affiliated Hospital of Nanjing Medical University, Nanjing \\ 210029, China. Email: bingrong.2002@163.com.
}

Background: Merkel cell carcinoma (MCC) is a rare and aggressive neuroendocrine carcinoma of the skin, with a high recurrence rate and a high mortality rate worldwide. The purpose of this article is to construct a nomogram that incorporates significant clinical parameters and predicts the survival of individuals with MCC.

Methods: The Surveillance, Epidemiology, and End Results (SEER) database was employed to retrospectively analyze all confirmed MCC cases from 2004 to 2015. The data was collected from 3,688 patients, and was randomized as the training or validation group (1:1 ratio). The independent factors which predicted the cancer-specific survival (CSS) and overall survival (OS) for MCC cases were searched for nomogram construction respectively. Independent parameters that affected CSS were determined using the Fine and Gray competing risk regression model. In addition, the time-dependent receiver operating characteristic (ROC) curve was constructed. Then, the area under the curve (AUC) values, calibration curve, and the concordance index (C-index) were used to determine the nomogram performance. At last, decision curve analysis (DCA) was conducted to determine the net clinical benefit.

Results: The multivariate analysis results revealed that sex, age, race, marriage, American Joint Committee on Cancer (AJCC) stage, chemotherapy and radiotherapy were independent OS prognostic factors. Furthermore, competing risk analysis showed age, sex, AJCC stage, chemotherapy were the independent CSS prognostic factors. For validation, the C-index value of OS nomogram was 0.703 (95\% CI: 0.686-0.721), while C-index value of CSS nomogram was 0.737 (95\% CI: 0.710-0.764). Both C-index and AUC suggested that nomograms had superior performance to that of the AJCC stage system. In addition, according to the calibration curve, both nomograms were capable of accurate prediction of MCC prognosis. The DCA showed that the net benefits of the nomograms were superior among various threshold probabilities than these of AJCC stage system.

Conclusions: The present work established and verified the novel nomograms to predict the OS and CSS of MCC patients. If further confirmed in future studies, it may become another helpful tool for risk stratification and management of MCC patients.

Keywords: Merkel cell carcinoma (MCC); nomogram; prognosis; survival

Submitted Jun 09, 2020. Accepted for publication Nov 26, 2020.

doi: $10.21037 /$ atm-20-4578

View this article at: http://dx.doi.org/10.21037/atm-20-4578

^ ORCID: 0000-0001-8156-9087. 


\section{Introduction}

Merkel cell carcinoma (MCC) represents an uncommon yet highly aggressive cutaneous neuroendocrine carcinoma. Its etiology and pathogenesis are mainly related to longterm excessive ultraviolet radiation exposure, together with infection with Merkel cell polyoma virus (1). In the elderly, MCC is frequently diagnosed in areas of sun-exposed skin and grows rapidly in these areas (2). In recent years, its incidence rate and mortality has significantly increased $(3,4)$, which is expected to be approximately 3,284 cases per year in 2025 in USA (3).

For clinicians, it is both an important and a difficult task to predict MCC prognosis and advising reasonable treatment options. At present, the American Joint Committee on Cancer (AJCC) stage classification has been identified as an effective tool to define the prognosis of MCC and to suggest the tailored treatment options to patients. AJCC classification has long exerted a vital part in evaluating the tumor prognosis $(5,6)$. The tumor stage classification system has been utilized for dividing patients into different risk groups, thus facilitating the assessment of clinical risks and the decision-making of clinical therapeutic strategy. However, the system has several limitations since only tumor size, histological metastasis and lymph nodes are considered $(7,8)$. Some researchers have indicated that even within the same tumor node metastasis (TNM) stage, the survival of MCC patients may differ $(9,10)$. While some other researchers suggested that the non-adjustment for sex- and age-matched life span in the overall survival (OS) possibly affected the creditability of this system (11). In addition, it is also reported that sex, ethnicity, the age of diagnosis, marital status, surgical interventions, adjuvant chemotherapy and radiotherapy significantly affect the survival of MCC patients. The AJCC classification system has been extensively adopted to predict patient prognosis. It is both important and difficult for clinicians to predict the prognosis of MCC and advise reasonable treatment options. After the possible AJCC classification system-based analysis was completed, we tried to develop effective prediction tools based on the existing stage systems, which will enable the early diagnosis of patients, and predict clinical outcomes and personalized treatments.

Nomograms are the graph-based representation of the results of the statistical prediction model, which makes it possible to quantify the prediction probability of clinical events of each patient (12). Nomograms are reliable and convenient tools for identifying positive and negative prognostic factors, which have been widely used in clinical practice to successfully predict the survival rate of cutaneous malignancies, like malignant melanoma and squamous cell carcinoma $(13,14)$.

As far as we know, there has been seldom study about nomograms in MCC prognosis prediction. This work aimed to establish and validate the nomograms based on the Surveillance, Epidemiology, and End Results (SEER) database, and incorporated various prognostic factors, which determined how diverse parameters affected the survival rate and visualized outcomes predicting individual patient survival. We presented the following article in accordance with the TRIPOD reporting checklist (available at http:// dx.doi.org/10.21037/atm-20-4578).

\section{Methods}

\section{Data source as well as inclusion criteria}

In this study, the latest version of SEER (8.3.5, https:// SEER.cancer.gov/) was applied in reviewing patient information in SEER database (covering 18 registries). The study was conducted in accordance with the Declaration of Helsinki (as revised in 2013). Since individual information has been removed from all the SEER databases, informed consent from patients and approval by the institutional review board were exempted. The patient inclusion criteria were as follows: (I) age $>18$ years; (II) all data contained ICD-O-3 histologic type (8247/3), and primary site code (44.0-44.9) was used for MCC; (III) all data had been classified by the New 8th Edition AJCC stage System; (IV) active follow-up with stage, surgery, tumor size, lymph node, and marriage; (V) patients in the cohort confirmed from 2004 to 2015. Patients conforming to the following conditions were excluded: (I) unknown stage information; (II) unknown surgery information; (III) unknown tumor size; (IV) unknown lymph involvement information; (V) unknown ethnic information; (VI) no information about marriage at diagnosis; (VII) primary site was not on skin. After selection, 3,688 eligible patients were included in analyses (flow diagram was shown in Figure 1). Patient demographic variables included age, sex, race, and marriage. In addition, the pathological stage (T/N/M, derived AJCC, eighth edition) of patients was included. The size of tumor was categorized into $\geq 2$ or $<2 \mathrm{~cm}$. Lymph nodes were classified as lymph involved or no lymph involved. Treatments included surgery, radiotherapy, and chemotherapy, which were further classified into two 


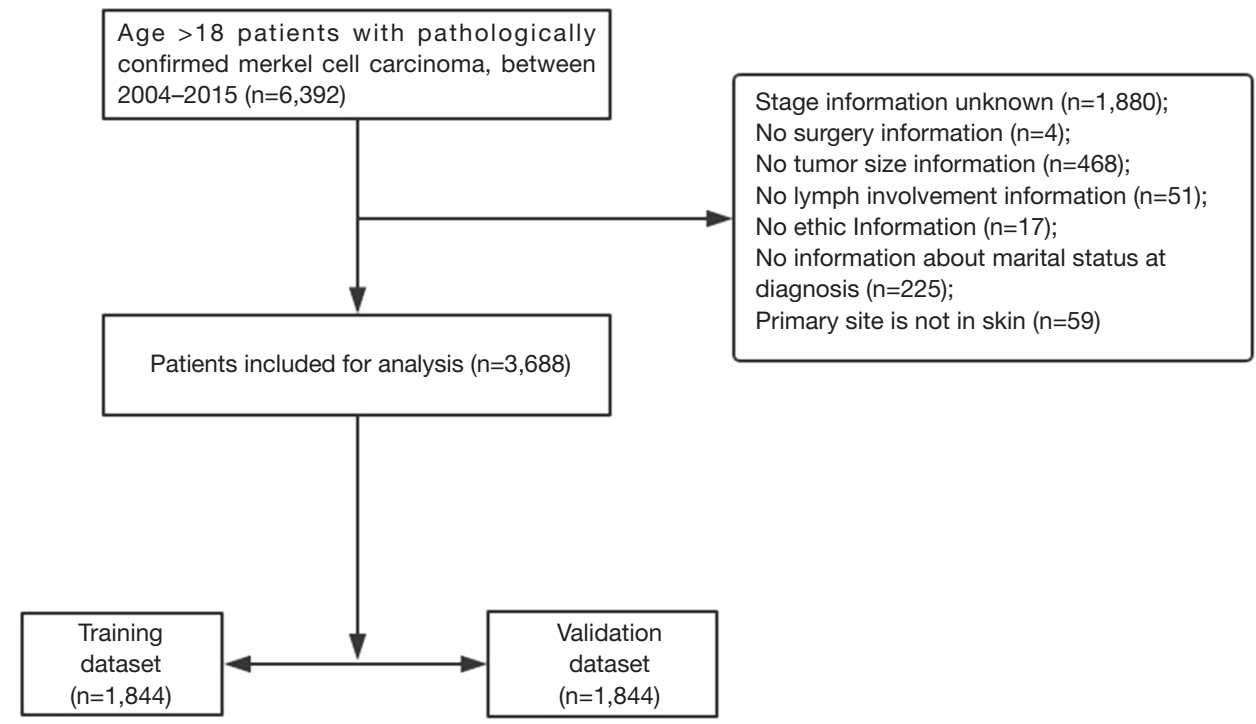

Figure 1 The flowchart of applying the inclusion and exclusion criteria and constructing training and validation dataset in the SEER database.

categories: "yes" and "no/unknown".

\section{Statistical analysis and nomogram construction}

The entire whole in the present work was randomized as two groups, including $1,844(50 \%)$ in the training cohort while $1,844(50 \%)$ in the validation cohort. At diagnosis, the age of a patient and survival time (months) were expressed as mean \pm SD or median and distributed appropriately (25th-75th percentile). Continuous variables were analyzed by Mann-Whitney test, whereas categorical variables were compared by chi-square test. Univariate and multivariate Cox proportional hazards regression was performed to assess factors related to patient survival.

The primary endpoint in our research was 3-year cancer-specific survival (CSS). The CSS was calculated after classification according to MCC characteristics, especially referring to people who die of MCC. Death due to additional causes may be the competitive event for MCC death. Therefore, the competing risk nomogram was built for predicting CSS (time period between MCC diagnosis and MCC-related death). Cumulative incidence function (CIF) depicted the accumulated occurrence probability of a certain event, when the competing events were also taken into consideration (15). The accumulated incidences of the interested events were fitted through designing the FineGray model (16).

In univariate analysis, the variables related to CSS $(\mathrm{P}<0.2)$ were further assessed with a multivariate Cox regression analysis based on proportional subdistribution hazard models. The independent variables with important clinical value were used to construct the nomogram of CSS.

The secondary endpoint in our research was 3 -year OS. OS represented the duration from the date of diagnosis to the date of death or last follow-up. In multivariate analysis, we included variables based on the results of the univariate analyses $(\mathrm{P}<0.2)$. The independent factors related to the prognosis of OS were determined using the multivariate Cox proportional hazards regression model. The CSS is the most relevant indicator, but OS should not be ignored.

The ROC curve was analyzed by determining the asconstructed nomogram performance. The nomogram accuracy was analyzed by Harrell C-index, together with the area under the time-dependent ROC curve (AUC). A greater $\mathrm{C}$-index value indicated the better nomogram performance in predicting the prognosis (17). A calibration curve of the nomograph was drawn to evaluate the consistency between the observed and estimated survivals. To sum up, the nomograms were constructed to predict the survivals for MCC patients at 3 years. We determined the clinical significance of using nomograms by performing decision curve analysis (DCA) (18). In 2008, Vickers extended the application scope of DCA to the analysis of survival data (19). For all analyses, a $\mathrm{P}$ value $<0.05$ indicated statistical significance. Statistical package R (http://www. r-project.org), together with EmpowerStats software 
(www.empowerstats.com, X\&Y Solutions, Inc., Boston, MA) was employed for statistical analysis.

\section{Results}

\section{The characteristics of patients}

According to patient inclusion as well as exclusion criteria, this study included 3,688 cases in the analyses. Then, all cases were randomized as the training $(n=1,844)$ or the validation $(n=1,844)$ cohort. There was no statistical difference among these cohorts. For all cases, their average age was 74.98 years, with the median survival of 28.00 months. In the present study, we included 2,337 (63.37\%) male patients. A majority of patients were white (95.72\%) in this study. The classification according to AJCC stages was as follows: stage I, 1,640 patients (44.47\%); stage II, 699 patients (18.95\%); stage III, 1,103 patients (29.91\%); and stage IV, 246 patients (6.67\%). With regard to therapy, most of the patients underwent surgery $(89.91 \%)$ and only 464 patients received chemotherapy (12.58\%). A total of 2,010 death cases were reported in the followup visits, including 645 cancer-related death cases as well as 1365 competing death cases. Table 1 displayed the clinicopathologic characteristics of all patients. Across the entire study population, the 3-year cancer-specific death rates of AJCC I, II, III and IV stages were 8.15\%, 12.29\%, $25.36 \%$ and $48.17 \%$, respectively; while the corresponding non-cancer-specific death rates were $25.48 \%, 32.22 \%$, $27.93 \%$ and $38.61 \%$. Figure S1 showed related CIF curves and the accumulated non-cancer-specific mortality increased relative to the cancer-specific mortality among cases in addition to AJCC IV stage.

\section{Nomogram establishment and verification in OS prediction}

To further determine the independent OS predicting factors, univariate together with multivariate analysis was conducted using the Cox proportional hazard model. According to results of univariate analysis, age, sex, marriage, tumor size, AJCC stage, lymph nodes, radiation, chemotherapy, and surgery were correlated with OS $(\mathrm{P}<0.05$, Table 2). By performing multivariate analysis, we identified seven independent prognostic factors of survival, such as sex, age, marriage, race, AJCC stage, radiotherapy, and chemotherapy (the nomogram is shown in Figure 2). From the nomogram, the age, which scale fell in the range of $0-100$, contributed to the OS at 3 years most significantly.
To apply the nomogram, drawing a perpendicular line towards the first row identify the value of each independent factors. The summation of the above figures was on the total-point axis, then the survival likelihood was determined by drawing a line down along the survival axis.

The C-index values for the as-constructed OS nomogram model in the training and validation set were 0.708 (95\% CI: 0.691-0.725) together with 0.703 (95\% CI: $0.686-$ 0.721 ), respectively, which had superior discriminating effect to those of AJCC stage within the above two sets (0.618, 95\% CI: $0.600-0.636)$ as well as $(0.610,95 \%$ CI: 0.592-0.628), respectively (Table 3). The AUC values of the ROC training and validation data predicted that our constructed nomogram had superior OS at 3 years to that of AJCC stage (Figure 3 and Table 3).

\section{Nomogram establishment and verification in CSS prediction}

Univariate and multivariate analysis results are displayed in Table 4. Upon univariate analysis, age, sex, tumor size, AJCC stage, chemotherapy, surgery, and lymph nodes were correlated with CSS $(\mathrm{P}<0.05)$. Besides, according to Fine and Gray analysis results, the age, sex, chemotherapy and AJCC stage were able to independently predict the prognosis for CSS (Figure 4). These four factors were used to construct the nomogram of CSS. Also, based on our constructed nomogram, the AJCC stage contributed most significantly to CSS (including 3-year).

With regard to the CSS nomogram model established, the C-index value for training set 0.745 (95\% CI: 0.719 0.771), while that for validation set was 0.737 (95\% CI: 0.710-0.764), which displayed superior discriminating effect to AJCC stage in either training $(0.715,95 \% \mathrm{CI}$ : $0.688-0.743)$ or validation $(0.700,95 \%$ CI: $0.671-0.730)$ set, respectively (Table 3). As shown in Figure 3, the AUC values of the training and validation data predicted that our constructed nomogram had superior 3-year CSS to AJCC stage.

\section{Calibration curve of the nomogram}

The calibration curves showed that the line of prediction closely approximated the 45-degree line, which presented the estimated survival rates in both training and validation sets at 3 years, respectively. These findings well conformed to the actual result, indicating an excellent agreement between OS and CSS at 3 years (Figures S2,S3). 
Table 1 Basic characteristics of the patients

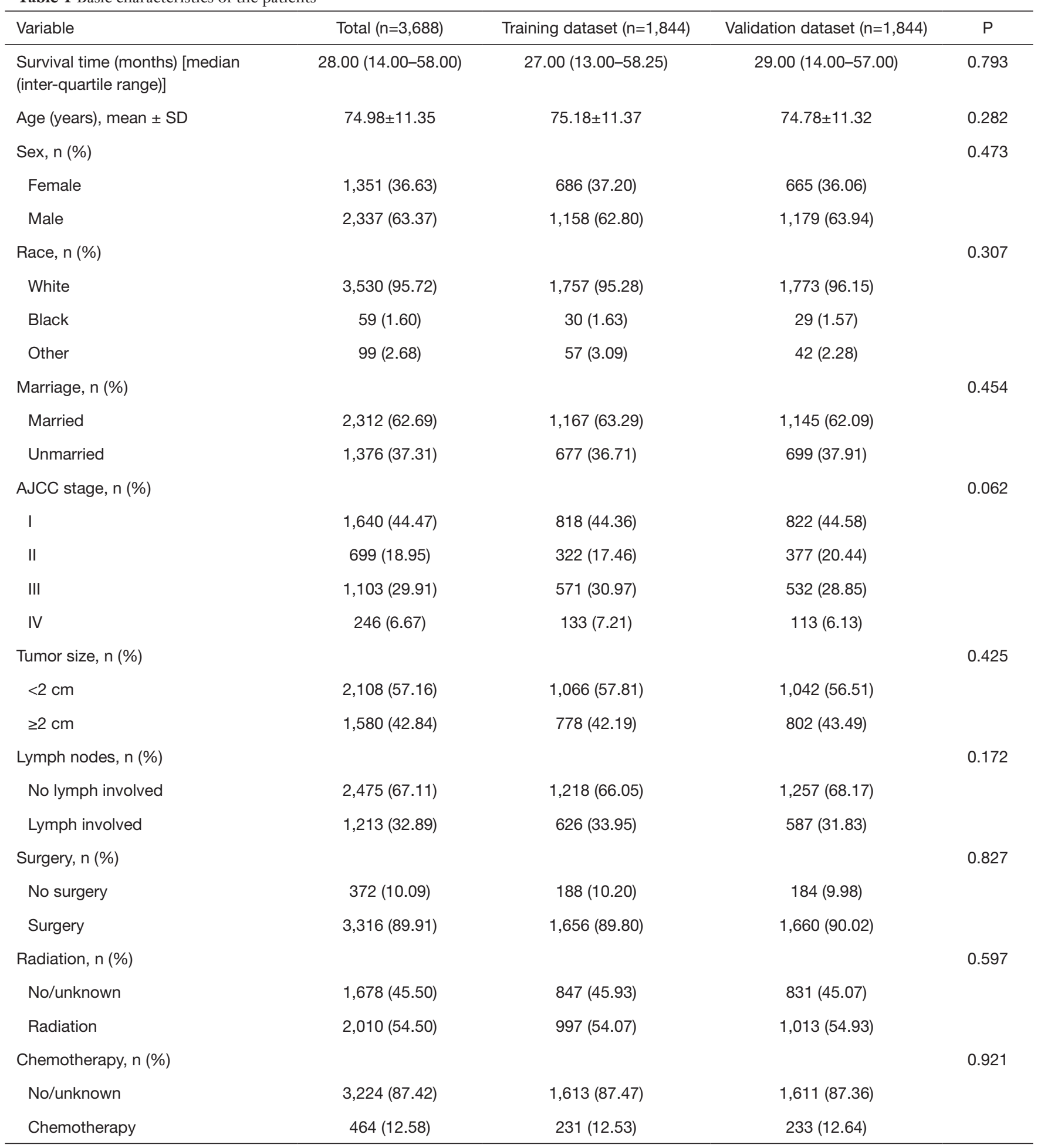

AJCC, American Joint Committee on Cancer; Cl, confidence interval. 
Page 6 of 13

Yin et al. Nomogram prediction for MCC

Table 2 Cox regression analysis for overall survival in the training dataset

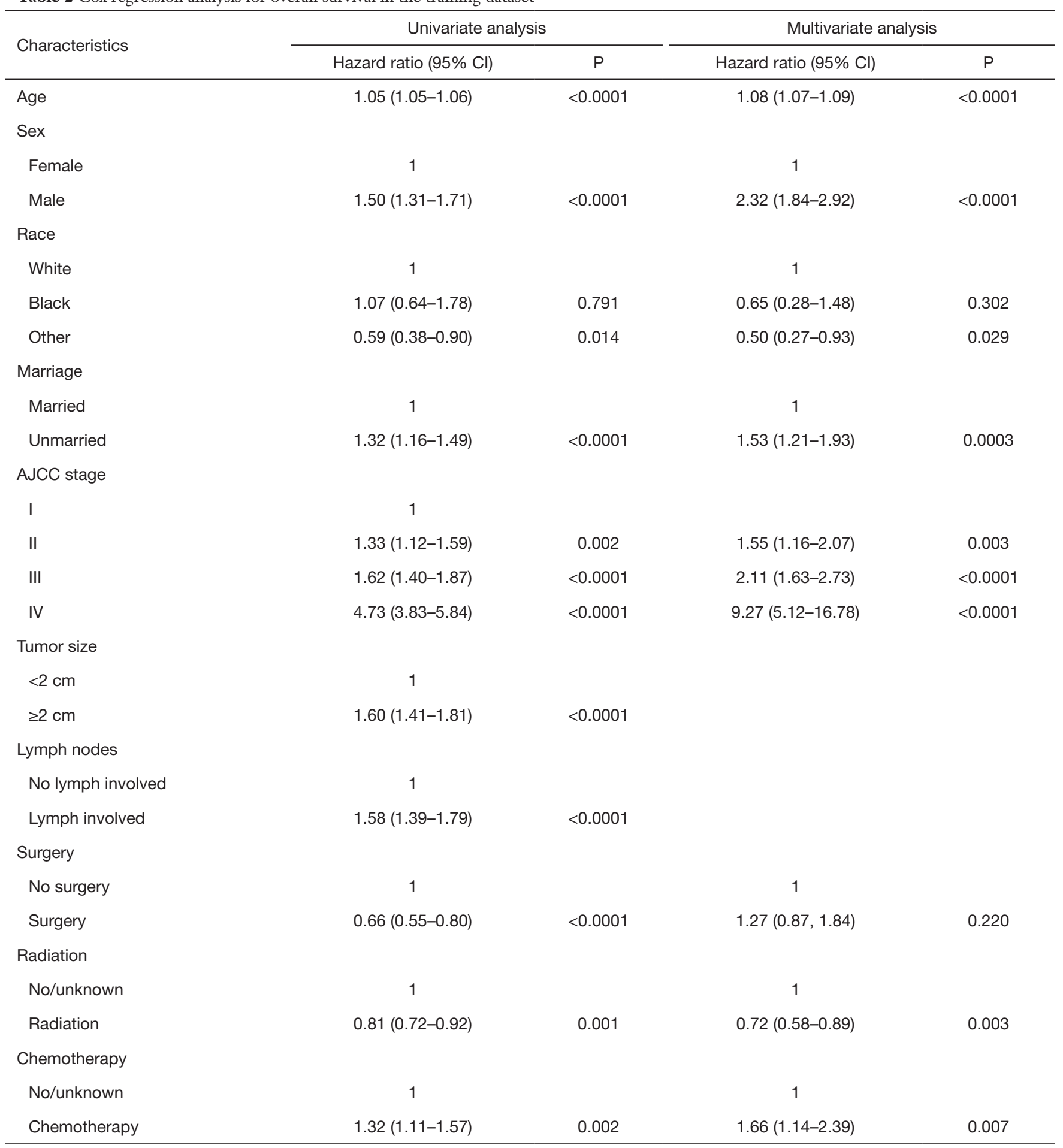




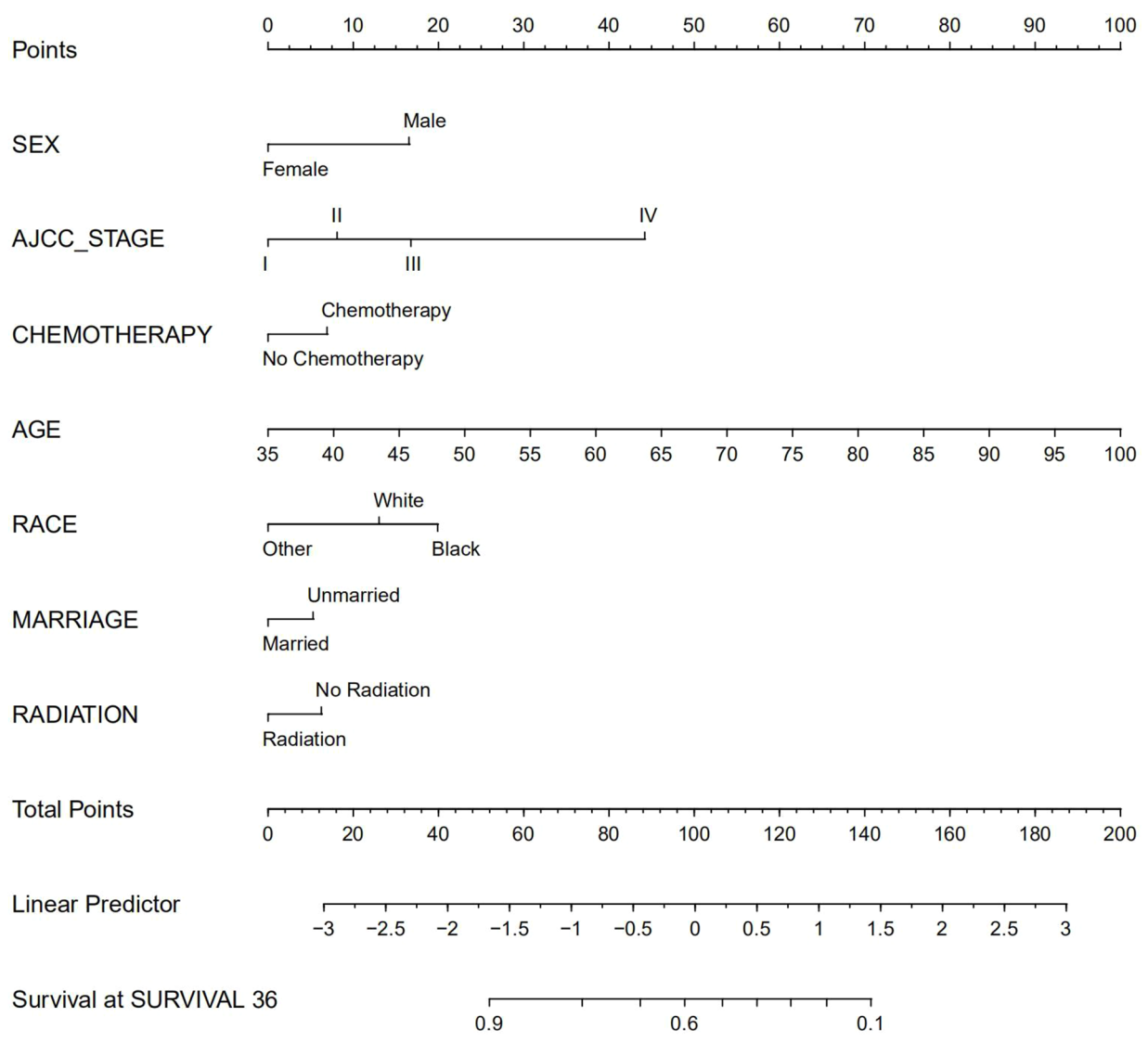

Figure 2 Nomogram predicting 3-year OS of the MCC patients. OS, overall survival; MCC, Merkel cell carcinoma.

\section{$D C A$}

Finally, DCA was adopted for assessing the nomogram clinical significance (Figures S4,S5). DCA, the integrative approach to estimate and compare nomogram with AJCC stage, can be established through calculating net benefits for diverse threshold likelihoods (20). To calculate net benefit, the false positive proportion was taken away from the true positive proportion, which was then weighted based on the relative hazard of the previous treatment relative to negative outcomes for the redundant treatment (19). Clinicians could decide whether interventions should be taken to the disease according the model whose performance was better.

\section{Discussion}

The mortality rate of MCC is high, and its incidence is growing faster than all solid tumors, even above that of malignant melanoma in the United States since the year 2000 (3). In this study, significant clinical parameters were used for establishing nomograms, which were used for predicting OS and CSS. As discovered on the basis of SEER database, sex, age, marriage, race, radiotherapy, surgery, chemotherapy and AJCC stage were independent factors affecting OS. Moreover, age, sex, chemotherapy and AJCC stage were identified to be the independent parameters affecting CSS. The results of AUC value, C-index, calibration curve, and DCA indicated that the predicted values of our nomograms were better than the AJCC stage.

Agreed well with those of previous study, in our nomograms, both OS and CSS were primarily affected by age and sex (21). Surgical treatment is the primary therapeutic option to treat MCC, especially in the case of primary MCC (22). We showed that surgery does not significantly improve the prognosis in patients. Some recent studies have showed conflicting results between different types of surgical interventions (22). Recurrence rate is up to $36-89 \%$ after surgical resection alone $(23,24)$. Further investigations are needed to resolve the affect of surgical interventions on MCC by randomized controlled trials. 
Table 3 Univariate and multivariate competing analysis of cancer specific survival in training set

\begin{tabular}{|c|c|c|c|c|}
\hline Characteristics & \multicolumn{2}{|c|}{ Univariate analysis } & \multicolumn{2}{|l|}{ Multivariate analysis } \\
\hline Age & $1.01(1.00-1.02)$ & 0.017 & $1.02(1.01-1.03)$ & 0.0003 \\
\hline \multicolumn{5}{|l|}{ Sex } \\
\hline Female & 1 & & 1 & \\
\hline \multicolumn{5}{|l|}{ Race } \\
\hline White & 1 & & & \\
\hline Black & $1.25(0.56-2.79)$ & 0.594 & $1.29(0.57-2.91)$ & 0.544 \\
\hline Other & $0.51(0.23-1.14)$ & 0.101 & $0.43(0.18-1.03)$ & 0.059 \\
\hline Unmarried & $1.17(0.94-1.46)$ & 0.152 & $1.19(0.95-1.51)$ & 0.137 \\
\hline \multicolumn{5}{|l|}{ AJCC stage } \\
\hline I & 1 & & & \\
\hline II & $1.76(1.22-2.53)$ & 0.003 & $1.71(1.18-2.47)$ & 0.005 \\
\hline III & $3.66(2.78-4.82)$ & $<0.0001$ & 3.44 (2.58-4.59) & $<0.0001$ \\
\hline IV & 13.05 (9.29-18.34) & $<0.0001$ & $11.42(7.77-16.79)$ & $<0.0001$ \\
\hline \multicolumn{5}{|l|}{ Tumor size } \\
\hline$<2 \mathrm{~cm}$ & 1 & & & \\
\hline No surgery & 1 & & 1 & \\
\hline Surgery & $0.51(0.38-0.68)$ & $<0.0001$ & $1.09(0.79-1.51)$ & 0.606 \\
\hline \multicolumn{5}{|l|}{ Radiation } \\
\hline No/unknown & 1 & & & \\
\hline Radiation & $1.08(0.87-1.34)$ & 0.473 & & \\
\hline \multicolumn{5}{|l|}{ Chemotherapy } \\
\hline No/unknown & 1 & & 1 & \\
\hline Chemotherapy & $2.72(2.13-3.47)$ & $<0.0001$ & 1.45 (1.08-1.94) & 0.013 \\
\hline
\end{tabular}

AJCC, American Joint Committee on Cancer; Cl, confidence interval. 


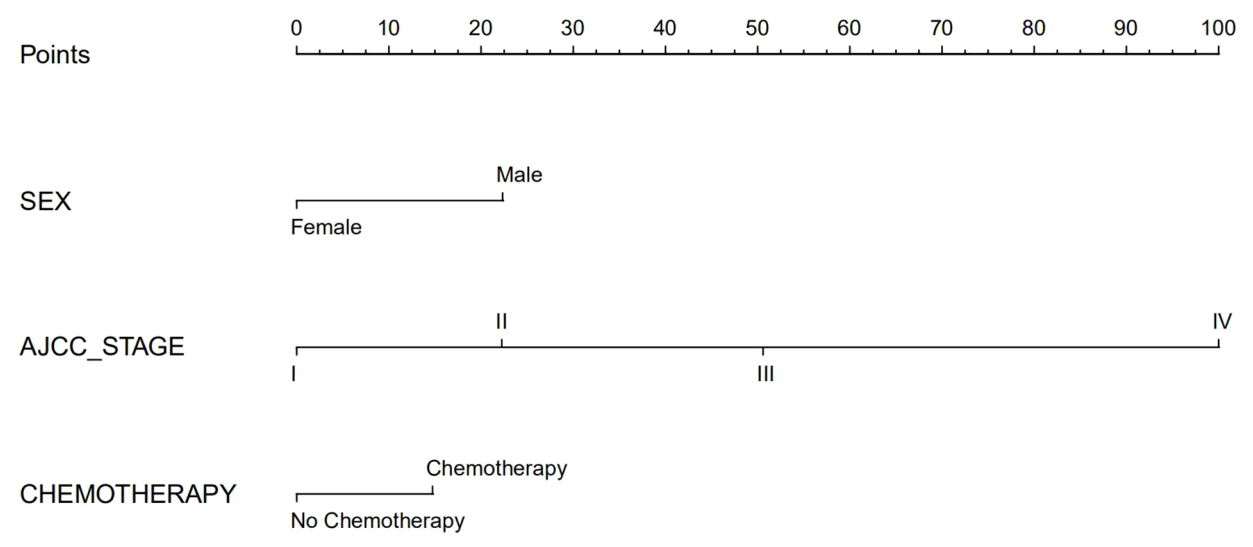

AGE

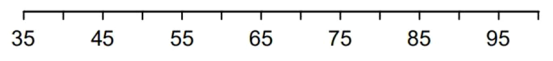

Total Points

\begin{tabular}{lllllllllll}
\hline 0 & 20 & 40 & 60 & 80 & 100 & 120 & 140 & 160 & 180 & 200
\end{tabular}

Linear Predictor

\begin{tabular}{lllllllllll}
\hline-2 & -1.5 & -1 & -0.5 & 0 & 0.5 & 1 & 1.5 & 2 & 2.5 & 3
\end{tabular}

Survival at SURVIVAL 36

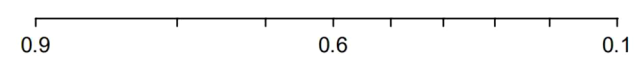

Figure 3 ROC analysis was performed to evaluate the AUC between the nomogram and AJCC stage. The training dataset (A) and validation dataset (B) of the OS. The training dataset (C) and validation dataset (D) of the CSS. ROC, receiver operating characteristic curve; AUC, area under receiver operating characteristic curve; AJCC, American Joint Committee on Cancer; OS, overall survival; CSS, cancer-specific survival.

Radiotherapy can be used to improve the prognosis of MCC patients. This is because MCC patients have high radiosensitivity (25-27). Radiotherapy is not only used as an alternative to radical surgical resection, but also as an adjuvant after surgery to improve the prognosis of patients $(25,28)$. These findings agreed well with our result that radiotherapy could improve the OS of MCC patients.

Chemotherapy is often used to improve the prognosis of MCC patients with lymph node metastases (28). In our analysis, chemotherapy had negative prognostic impact, reducing the 3 -year specific survival rate. This is because most patients were treated with traditional chemotherapy regimens. Currently, the traditional chemotherapy regimen for MCC is derived from small cell lung cancer, and the first-line chemotherapy is mainly etoposide combined with carboplatin or cisplatin $(29,30)$. In addition, chemotherapeutic drugs are cytotoxic, causing pain and relatively high mortality in patients (31). Nevertheless, future clinical trials should continue to explore the role of chemotherapy in MCC patients.

Unmarried status was independent adverse prognostic factors for MCC. Some studies suggest that married person could get more social and economic support (32). In addition, the spousal detection of MCC could promote early diagnosis and treatment in skin tumors (33). However, these prognostic factors were not identified very significantly in the nomograms of OS and CSS at the same time. Furthermore, Kim et al. reported that an improvement in OS was associated with difference or bias in those unmeasured parameters, and the hidden bias might greatly change those results (27).

There are some advantages to our study. Firstly, the SEER database was used in this study, which included a huge American population. Altogether, 3,688 MCC patients 
Page 10 of 13

Yin et al. Nomogram prediction for MCC

Table 4 Comparison of C-indexes and AUC between the nomograms and AJCC stages in MCC patients

\begin{tabular}{|c|c|c|c|c|c|c|c|c|}
\hline Survival types & \multicolumn{4}{|c|}{ Training set } & \multicolumn{4}{|c|}{ Validation set } \\
\hline \multicolumn{9}{|l|}{ OS } \\
\hline Nomogram & 0.708 & $0.691-0.725$ & 0.762 & $0.741-0.784$ & 0.703 & $0.686-0.721$ & 0.765 & $0.744-0.786$ \\
\hline AJCC stage & 0.618 & $0.600-0.636$ & 0.604 & $0.580-0.628$ & 0.61 & $0.592-0.628$ & 0.611 & $0.587-0.635$ \\
\hline \multicolumn{9}{|l|}{ CSS } \\
\hline Nomogram & 0.745 & $0.719-0.771$ & 0.715 & $0.686-0.744$ & 0.737 & $0.710-0.764$ & 0.711 & $0.679-0.743$ \\
\hline AJCC stage & 0.715 & $0.688-0.743$ & 0.689 & $0.659-0.719$ & 0.7 & $0.671-0.730$ & 0.683 & $0.651-0.716$ \\
\hline
\end{tabular}

AJCC, American Joint Committee on Cancer; AUC, area under curve; Cl, confidence interval; OS, overall survival; CSS, cancer specific survival.
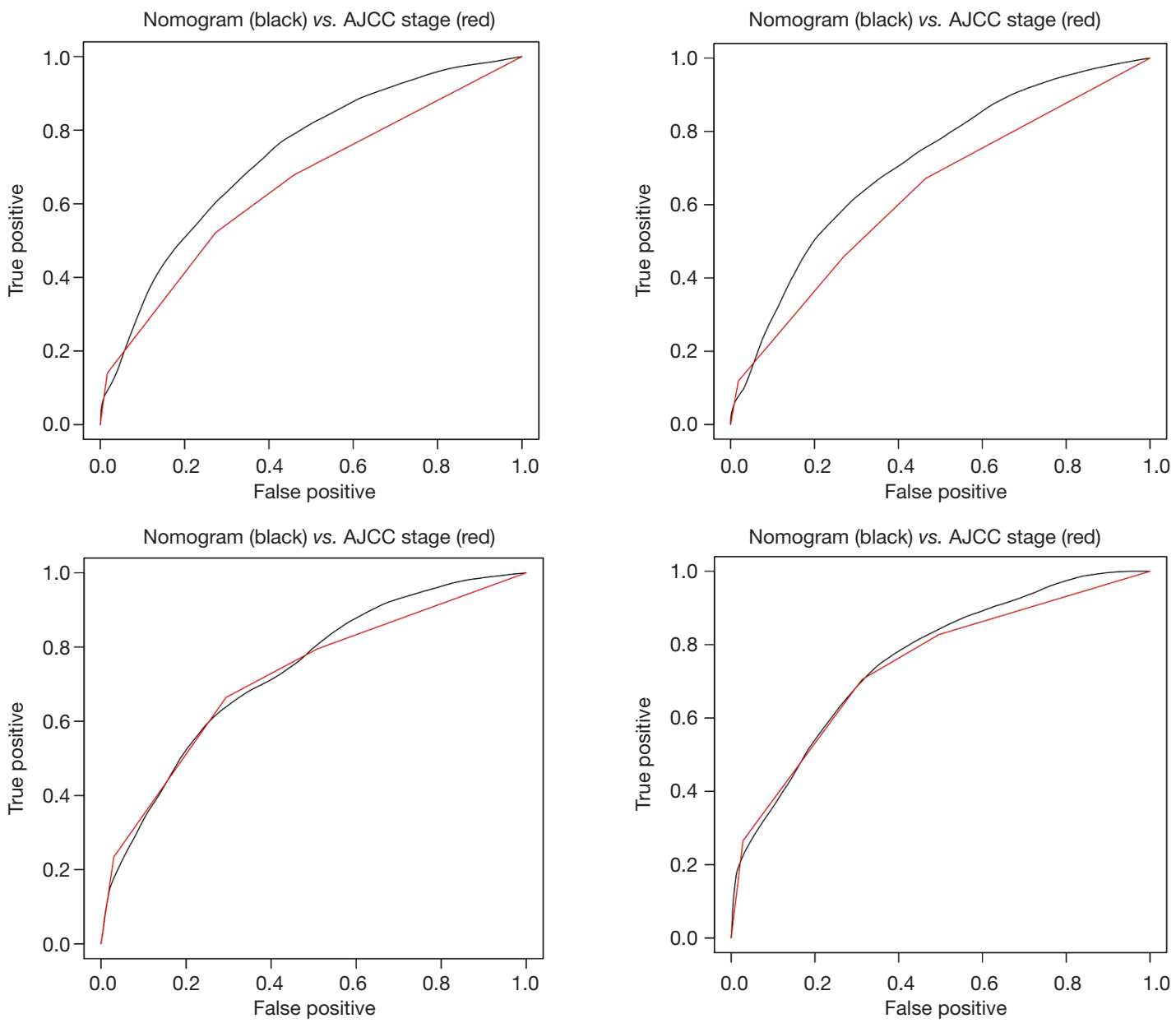

Figure 4 Nomogram predicting 3-year CSS of the MCC patients. CSS, cancer-specific survival; MCC, Merkel cell carcinoma. 
complied with the standards, and they were screened out of 6,392 patients. Secondly, many characteristics are included in our analysis, such as demographic characteristics, AJCC stage, and treatment strategies. As a result, our nomograms have good generalizability and do not need to be limited by sex, race, and marriage. Finally, the predictive performance and the accuracy of our nomograms was better than those of AJCC stage. Moreover, our nomograms were tested by ROC analysis, calibration curve, and DCA, to predict the survival of MCC patients.

Our study also has limitations. Firstly, the majority patients from SEER database were white race, so there was potential racial heterogeneity that could not be extrapolated to other human species. Secondly, the variables obtained from SEER database were limited, and there was some important variable information that was not available, including the "No/Unknown" information, type, or extent of treatment and specific doses and drug regimens used. In addition, we did not account for length of time required for staging and treatment, which may lead to immortal time bias. We will collect data from multiple medical centers in different regions and at different levels in the future to optimize and validate the model. Finally, as the pathogenesis of the disease continues to be explored, and new treatment options continue to be improved, such as the application of targeted drugs like PD-1, PD-L1, the prognosis of patients will certainly change. The information data of this nomogram would also need to be updated. Notably, the predicted values were calculated by using only the nomogram, which is a kind of reference information and not an absolutely accurate prognosis.

\section{Conclusions}

The nomogram model, which was developed on the SEER database, provides a reliable and practical tool for assessing the OS as well as CSS for MCC, and a variety of independent parameters were integrated for prognosis prediction. Thus, it exhibited better discrimination and calibration than the traditional AJCC stage. However, we need an external validation method for further verifying our findings. If further validated by other studies, nomograms have the potential to become a useful clinical prediction tool for MCC.

\section{Acknowledgments}

The authors would like to thank SEER for open access to the database.

Funding: This work was supported by grants from the China National Natural Science Foundation (82073472), the Priority Academic Program Development (PAPD) of Jiangsu Higher Education Institutions and Outstanding Young and Middle-aged Talents Support Program of the First Affiliated Hospital with Nanjing Medical University (Jiangsu Province Hospital). BZ is funded by the Project of Key Youth Medical Talent of Jiangsu Province (QNNRC 2016583) and Outstanding Young Backbone Teachers of Nanjing Medical University (in 2017).

\section{Footnote}

Reporting Checklist: The authors have completed the TRIPOD reporting checklist. Available at http://dx.doi. org/10.21037/atm-20-4578

Peer Review File: Available at http://dx.doi.org/10.21037/ atm-20-4578

Conflicts of Interest: All authors have completed the ICMJE uniform disclosure form (available at http://dx.doi. org/10.21037/atm-20-4578). The authors have no conflicts of interest to declare.

Ethical Statement: The authors are accountable for all aspects of the work in ensuring that questions related to the accuracy or integrity of any part of the work are appropriately investigated and resolved. The study was conducted in accordance with the Declaration of Helsinki (as revised in 2013). Since individual information has been removed from all the SEER databases, informed consent from patients and approval by the institutional review board were exempted.

Open Access Statement: This is an Open Access article distributed in accordance with the Creative Commons Attribution-NonCommercial-NoDerivs 4.0 International License (CC BY-NC-ND 4.0), which permits the noncommercial replication and distribution of the article with the strict proviso that no changes or edits are made and the original work is properly cited (including links to both the formal publication through the relevant DOI and the license). See: https://creativecommons.org/licenses/by-nc-nd/4.0/.

\section{References}

1. Lemos B, Nghiem P. Merkel cell carcinoma: more 
deaths but still no pathway to blame. J Invest Dermatol 2007;127:2100-3.

2. Prewett SL, Ajithkumar T. Merkel Cell Carcinoma: Current Management and Controversies. Clin Oncol (R Coll Radiol) 2015;27:436-44.

3. Paulson KG, Park SY, Vandeven NA, et al. Merkel cell carcinoma: Current US incidence and projected increases based on changing demographics. J Am Acad Dermatol 2018;78:457-63.e2.

4. Fitzgerald TL, Dennis S, Kachare SD, et al. Dramatic Increase in the Incidence and Mortality from Merkel Cell Carcinoma in the United States. Am Surg 2015;81:802-6.

5. Dong F, Shen Y, Gao F, et al. Nomograms to Predict Individual Prognosis of Patients with Primary Small Cell Carcinoma of the Bladder. J Cancer 2018;9:1152-64.

6. $\mathrm{Pu} \mathrm{N}, \mathrm{Li} \mathrm{J}, \mathrm{Xu} \mathrm{Y}$, et al. Comparison of prognostic prediction between nomogram based on lymph node ratio and AJCC 8th staging system for patients with resected pancreatic head carcinoma: a SEER analysis. Cancer Manag Res 2018;10:227-38.

7. Kang JS, Lee S, Son D, et al. Prognostic predictability of the new American Joint Committee on Cancer 8th staging system for distal bile duct cancer: limited usefulness compared with the 7th staging system. J Hepatobiliary Pancreat Sci 2018;25:124-30.

8. Yang L, Wang S, Zhou Y, et al. Evaluation of the 7(th) and 8(th) editions of the AJCC/UICC TNM staging systems for lung cancer in a large North American cohort. Oncotarget 2017;8:66784-95.

9. Harms KL, Healy MA, Nghiem P, et al. Analysis of Prognostic Factors from 9387 Merkel Cell Carcinoma Cases Forms the Basis for the New 8th Edition AJCC Staging System. Ann Surg Oncol 2016;23:3564-71.

10. Farley CR, Perez MC, Soelling SJ, et al. Merkel Cell Carcinoma Outcomes: Does AJCC8 Underestimate Survival? Ann Surg Oncol 2020;27:1978-85.

11. Tothill R, Estall V, Rischin D. Merkel cell carcinoma: emerging biology, current approaches, and future directions. Am Soc Clin Oncol Educ Book 2015;e519-26.

12. Iasonos A, Schrag D, Raj GV, et al. How to build and interpret a nomogram for cancer prognosis. J Clin Oncol 2008;26:1364-70.

13. Yang J, Pan Z, Zhao F, et al. A nomogram for predicting survival in patients with nodular melanoma: A populationbased study. Medicine (Baltimore) 2019;98:e16059.

14. Levy DA, Li H, Sterba KR, et al. Development and Validation of Nomograms for Predicting Delayed Postoperative Radiotherapy Initiation in Head and Neck
Squamous Cell Carcinoma. JAMA Otolaryngol Head Neck Surg 2020;146:455-64.

15. Austin PC, Fine JP. Practical recommendations for reporting Fine-Gray model analyses for competing risk data. Stat Med 2017;36:4391-400.

16. Berger M, Schmid M, Welchowski T, et al. Subdistribution hazard models for competing risks in discrete time. Biostatistics 2020;21:449-66.

17. Huitzil-Melendez FD, Capanu M, O'Reilly EM, et al. Advanced hepatocellular carcinoma: which staging systems best predict prognosis? J Clin Oncol 2010;28:2889-95.

18. $\mathrm{Zi} \mathrm{H}, \mathrm{Gao} \mathrm{L}, \mathrm{Yu} Z$, et al. Nomograms for predicting long-term overall survival and cancer-specific survival in patients with primary urethral carcinoma: a populationbased study. Int Urol Nephrol 2020;52:287-300.

19. Vickers AJ, Cronin AM, Elkin EB, et al. Extensions to decision curve analysis, a novel method for evaluating diagnostic tests, prediction models and molecular markers. BMC Med Inform Decis Mak 2008;8:53.

20. Vickers AJ, Elkin EB: Decision curve analysis: a novel method for evaluating prediction models. Med Decis Making 2006;26:565-74.

21. Robertson JP, Liang ES, Martin RC. Epidemiology of Merkel cell carcinoma in New Zealand: a populationbased study. Br J Dermatol 2015;173:835-7.

22. Yan L, Sun L, Guan Z, et al. Analysis of cutaneous Merkel Cell Carcinoma outcomes after different surgical interventions. J Am Acad Dermatol 2020;82:1422-34.

23. Morrison WH, Peters LJ, Silva EG, et al. The essential role of radiation therapy in securing locoregional control of Merkel cell carcinoma. Int J Radiat Oncol Biol Phys 1990;19:583-91.

24. Eich HT, Eich D, Staar S, et al. Role of postoperative radiotherapy in the management of Merkel cell carcinoma. Am J Clin Oncol 2002;25:50-6.

25. Wright GP, Holtzman MP. Surgical resection improves median overall survival with marginal improvement in long-term survival when compared with definitive radiotherapy in Merkel cell carcinoma: A propensity score matched analysis of the National Cancer Database. Am J Surg 2018;215:384-7.

26. Leonard JH, Ramsay JR, Kearsley JH, et al. Radiation sensitivity of Merkel cell carcinoma cell lines. Int J Radiat Oncol Biol Phys 1995;32:1401-7.

27. Kim JA, Choi AH. Effect of Radiation Therapy on Survival in Patients With Resected Merkel Cell Carcinoma. JAMA Dermatol 2013;149:831.

28. Bhatia S, Storer BE, Iyer JG, et al. Adjuvant Radiation 
Therapy and Chemotherapy in Merkel Cell Carcinoma: Survival Analyses of 6908 Cases From the National Cancer Data Base. J Natl Cancer Inst 2016;108:djw042.

29. Steven N, Lawton P, Poulsen M. Merkel Cell Carcinoma Current Controversies and Future Directions. Clin Oncol (R Coll Radiol) 2019;31:789-96.

30. Iyer JG, Blom A, Doumani R, et al. Response rates and durability of chemotherapy among 62 patients with metastatic Merkel cell carcinoma. Cancer Med 2016;5:2294-301.

31. Voog E, Biron P, Martin JP, et al. Chemotherapy for

Cite this article as: Yin X, She H, Martin Kasyanju Carrero L, $\mathrm{Ma}$ W, Zhou B. Nomogram prediction for the overall survival and cancer-specific survival of patients diagnosed with Merkel cell carcinoma. Ann Transl Med 2021;9(4):286. doi: 10.21037/ atm-20-4578 patients with locally advanced or metastatic Merkel cell carcinoma. Cancer 1999;85:2589-2595.

32. Liu MA, Nguyen J, Driver JA. Influence of age and marital status on stage at diagnosis and survival of patients with Merkel cell carcinoma: A Surveillance, Epidemiology, and End Results registry-based cohort study. J Am Acad Dermatol 2018;79:1146-8.

33. Reyes Ortiz CA, Freeman JL, Kuo YF, et al. The influence of marital status on stage at diagnosis and survival of older persons with melanoma. J Gerontol A Biol Sci Med Sci 2007;62:892-8. 